\title{
Quasi-Impulsive Maneuvers to Correct Mean Orbital Elements in LEO
}

\author{
Federico Fumenti, Markus Schlotterer and Stephan Theil
}

\begin{abstract}
An approach is developed to compute quasi-impulsive maneuvers to steer the orbital elements of a spacecraft to a desired value. Using Gauss Variational Equations it is possible to define the location along the orbit as well as the magnitude of the maneuver(s) so that specific orbital elements can be changed with little influence on the others. The possibility to include the effect of the zonal terms of the gravity model of the Earth and of the atmospheric drag allows an accurate evaluation of the time required to reach the maneuvering location. Including a model of the propulsion system makes the simulation more realistic, if compared with an impulsive maneuver implementation, since a burning arc can replace the istantaneous change of the orbital elements, which is instead associated with the impulsive approach. Simulations have been performed to compare perturbed and unperturbed cases and the results from the comparisons are presented.
\end{abstract}

\section{Introduction}

The majority of spacecraft, once deployed, do not follow a pure natural motion for their entire lifetime. Rather, every now and then they require an orbital maneuver in order to alter their state or, equivalently, their orbital elements. According to the mission requirements, different constraints can be imposed on the maneuvers concerning the direction of the thrust, the fuel consumption, the burning time, etc. When modeling a $\Delta V$, it can be decided to follow an impulsive or a continuous approach, depending whether the assumption of zero burning time can be held or not. As a matter of fact, when the burning time is short compared to the orbital period, the aforementioned assumption is valid and the application of the first approach is feasible. The simplicity associated with the computation of the required $\Delta V$ makes

Federico Fumenti, Markus Schlotterer, Stephan Theil

DLR, Institute of Space Systems, Robert Hooke Str. 7, 28359, Bremen

e-mail: federico.fumenti@dlr.de 
the impulsive approach widely used in the preliminary analysis of a transfer. Refs. $[5,6,8,9,10,16]$ are examples of investigations on the impulsive maneuvering approach. On the other side, no burning time assumptions are required to apply the continuous approach, but in this case the modeling of the transfer involves more complexity, being the required $\Delta V$ is spread along an arc of the trajectory. Studies on the continuous approach can be found in Refs. [11, 12, 13]. These works are particularly interesting because the authors evaluate the maneuvers exploiting the Gauss Variational Equations (GVE). The peculiarity of the GVE is that they show the effects of a perturbing force on the orbit elements of the spacecraft, regardless on the nature of the force, which can be natural (deriving from the geopotential or atmospheric drag effect) or artificial (due to the activation of a thruster). Using the GVE, Schaub \& Alfriend [14] developed an impulsive feedback control for establishing specific mean orbit elements for spacecraft in a formation flying environment, while requiring only 1 or 2 impulses. Beigelman \& Gurfil [3] dealt with the geostationary collocation problem and proposed an impulsive strategy based on the differences in the orbit elements and on the GVE. Similarly, Anderson \& Schaub [1] combined the knowledge of the differential orbit elements of 2 spacecraft with the GVE and developed an N-impulse feedback control strategy. As can be seen, the use of the GVE finds its perfect application in a coordinated flight mission, where multiple spacecraft are employed and relative distances must be respected. In this type of missions in fact, it is useful to express the relative motion in terms of relative orbital elements and use the mean orbital elements to have a clear overview of the long-term behaviour. In such a framework no large element changes are needed and the required maneuvers are only aimed at preserving the relative motion through applying small elements variations to counteract the effect of the perturbations (or more exactly, the differential effect of the perturbations). To evaluate the required control actions, the GVE become helpful.

The challenge associated with the GVE lies in the coupling of the equations, since the application of the thrust in one direction can affect more than one orbital element. On the other side, when this coupling is properly exploited an advantageous maneuver can be designed to change specific elements with theoretically no effect on the others.

In order to make the simulation more realistic, the knowledge of the propulsion system can be employed and a finite duration of the control actions can be evaluated. Nevertheless, the need for changing the orbital elements only by a small amount ensures the control actions to remain very short, which motivates the definition of quasi-impulsive maneuvers.

As mentioned above, the choice of using the impulsive or the finite approach depends mainly on the duration of the control action, but when this parameter is small it becomes harder to choose: on one side the impulsive approach is highly simple, on the other side the finite approach can lead to better results. In the quasiimpulsive approach, the problem to be solved has been based on the equations and approximations typically used in the impulsive approach in order to keep simplicity and ensure easy resolution. At the same time though, it has been extended to im- 
prove the fidelity of the simulation through including equations expressing the finite duration of the control actions.

In this work the authors dealt with maneuvers to change the semimajor axis (a), the eccentricity $(e)$ and the inclination $(i)$, without significantly affecting the other orbital elements, for a satellite in low Earth orbit (LEO). The intention of focusing only on those three elements has been dictated by the concept of the $J_{2}$-invariant orbits [15], which in recent years has been widely accepted and applied. At the basis of this method lies the assumption that all the spacecraft in a given formation are perturbed only by the $J_{2}$ term, then natural relative orbits can be analytically found matching the rates of the affected mean elements and finding the $(a, e, i)$ triplet for each agent, since the imposed constraints depend only on these three elements. This means that the relative motion can be controlled by only controlling 3 out of 6 elements, which in turn explains the interest of this paper.

Nonetheless, to increase the accuracy of the model, the effect of perturbations other than only $J_{2}$ have been considered and their inclusion has been discussed.

The paper is organised as follows. In Section 2 the problem is introduced along with the used models. The control strategy is presented in Section 3, while Section 4 deals with numerical simulations and presents single test examples as well as Monte-Carlo tests campaigns. Finally, conlusions are given in Section 5.

\section{Problem Statement}

When projecting a coordinated flight mission, with multiple spacecraft involved, a key role for the success of the whole mission is played by the relative motion among the agents; hence it is vital to develop strategies to control this feature. Let us consider for example the scenario described in [17] for a manned Mars mission. Due to the huge mass required, the plan for the deployment of the space station consists in building and launching several spacecraft at different times and perform an inorbit assembly to obtain the final structure. In such a framework, it looks unrealistic that each element is immediately assembled right after the launch. Rather, it is reasonable to assume that it will spend some time in orbit, with the outcome that for most of the time a certain number of spacecraft will fly together in a cluster. All the spacecraft will have similar orbital elements and will fly in the neighbourhood of a nominal orbit used as reference (it could be the orbit of a particular agent, the orbit of the cluster barycenter, a theoretical orbit, etc.).

In a likely scenario, the use of a low Earth orbit is envisaged with first mean orbital elements for the nominal orbit $(a, e, i)=(7000 \mathrm{~km}, 0.001,50 \mathrm{deg})$ (throughout the paper these values will be referred to as the nominal values). This means that only the gravitational potential of the Earth and the atmospheric drag significantly affect the motion of the satellites.

If only the $J_{2}$ term perturbation would be included, the use of the $J_{2}$-invariant orbits would be natural. In such a framework only the evolution of the right ascension of the ascending node (RAAN) $\Omega$, of the argument of perigee $\omega$ and of the mean 
anomaly $I H$ are affected, constraints to match the rates $\dot{\Omega}, \dot{\omega}$ and $\dot{H}$ of the different agents are imposed and natural relative orbits can be found. The initial values for $a, e$, and $i$ can be chosen in order to meet the constraints, while those for $\Omega, \omega$ and I $\mathrm{A}$ can be properly selected to respect the relative distances. Conversely, when other perturbations are included the aforementioned constraints based on the mean elements rates must be adapted and natural relative orbits are not available anymore. On the other side the effect of perturbations like higher order of the geopotential or atmospheric drag is much smaller than the effect of the $J_{2}$ term, so much that the application of the $J_{2}$-invariant orbits could still be feasible. The authors propose then to keep exploiting only the $J_{2}$ term to find the initial configuration, while a more accurate model can be employed to simulate the satellites motion and to evaluate the location of the maneuvers required to control $a, e$ and $i$, while minimizing the changes in $\Omega, \omega$ and $\pi$.

At this point it must be remembered that the $J_{2}$-invariant orbits guarantee all satellites having the same rate of change for the argument of latitude $(\theta)$, but nothing can be said for the argument of perigee or the mean anomaly. These two elements may still change at different rates causing a drifting of the lines of perigee and then a change in the geometry of the relative orbits. This means that proper actions must be taken to control $\omega$ and $/$, but they are not discussed in this work.

Let us suppose that at a certain time, an agent of the cluster requires a correction in its mean elements. To see the effect of a control vector on the osculating elements the GVE can be used. The following system shows the equations when the control action $\mathbf{u}=\left(\begin{array}{lll}u_{p} & u_{\theta} & u_{h}\end{array}\right)$ is given in the $S p \theta h$ orbital frame, where $S$ is the origin of the reference frame and is coincident with the satellite, the axis $p$ is aligned with the position vector, the axis $h$ is aligned with the angular momentum vector, the axis $\theta$ is consequently placed in order to have a right-hand oriented frame [2]:

$$
\begin{aligned}
\frac{d a}{d t} & =\frac{2 a^{2}}{h}\left(e \sin (\boldsymbol{A}) u_{p}+\frac{p}{r} u_{\theta}\right) \\
\frac{d e}{d t} & =\frac{1}{h}\left\{p \sin (\boldsymbol{A}) u_{p}+[(p+r) \cos (\boldsymbol{A})+r e] u_{\theta}\right\} \\
\frac{d i}{d t} & =\frac{r \cos \theta}{h} u_{h} \\
\frac{d \Omega}{d t} & =\frac{r \sin \theta}{h \sin i} u_{h} \\
\frac{d \omega}{d t} & =\frac{1}{h e}\left[-p \cos (\boldsymbol{A}) u_{p}+(p+r) \sin (\boldsymbol{A}) u_{\theta}\right]-\frac{r \sin \theta \cos i}{h \sin i} u_{h} \\
\frac{d r}{d t} & =n+\frac{\eta}{h e}\left[(p \cos (\boldsymbol{A})-2 r e) u_{p}-(p+r) \sin (\boldsymbol{A}) u_{\theta}\right]
\end{aligned}
$$

where $r$ is the orbital radius, $h$ the angular momentum, $p$ the semilatus rectum, 7 the true anomaly, $\eta=\sqrt{1-e^{2}}$ an additional eccentricity measure, $n=\sqrt{\frac{\mu}{a^{3}}}$ the mean motion and $\mu=398604.415 \mathrm{~km}^{2} / \mathrm{s}^{3}$ the gravitational parameter of the Earth. When the more compact matrix form is employed, the GVE can also be expressed 
by

$$
\dot{\mathbf{e}}=\left[\begin{array}{llllll}
0 & 0 & 0 & 0 & 0 & n
\end{array}\right]^{T}+B(\mathbf{e}) \mathbf{u}
$$

with $\mathbf{e}=(\text { a e i } \Omega \omega / 7)^{T}$ denoting the osculating orbital elements vector. As can be seen, the GVE are based on the osculating elements, hence to study the effect of $\mathbf{u}$ on the mean ones $\overline{\mathbf{e}}$, Eq. (1) or (2) must be coupled with a transformation

$$
\overline{\mathbf{e}}=f(\mathbf{e})
$$

which leads to

$$
\dot{\overline{\mathbf{e}}}=\left(\frac{\partial \overline{\mathbf{e}}}{\partial \mathbf{e}}\right)^{T} \frac{d \mathbf{e}}{d t} \approx \frac{d \mathbf{e}}{d t}=\left[\begin{array}{llllll}
0 & 0 & 0 & 0 & 0 & n
\end{array}\right]^{T}+B(\mathbf{e}) \mathbf{u} .
$$

In Eq. (4) the approximation derives from the transformation between osculating and mean elements taken from Brouwer's theory, which shows how the sensitivity matrix $\left(\frac{\partial \overline{\mathbf{e}}}{\partial \mathbf{e}}\right)^{T}$ is basically an identity matrix with the off-diagonal terms being $O\left(J_{2}\right)$ [13]. In other words, it can be assumed that when a control action is applied to change the generic element $\propto$, the effect on both the osculating and the mean values will be the same and they will change by the same amount.

Please note now that the GVE use the current elements of the satellite. In this work the authors assumed that a certain amount of time will pass between the computation and the application of a control action, then in the computation phase a prediction of the orbital elements values is required. Since predicting $\overline{\mathbf{e}}$ is much easier than predicting $\mathbf{e}$ and the difference between $B(\overline{\mathbf{e}})$ and $B(\mathbf{e})$ is negligible, it has been decided to introduce this second approximation and use the matrix $B(\overline{\mathbf{e}})$.

Finally, the matrix form of the GVE becomes

$$
\dot{\overline{\mathbf{e}}}=\left[\begin{array}{llllll}
0 & 0 & 0 & 0 & 0 & n
\end{array}\right]^{T}+B(\overline{\mathbf{e}}) \mathbf{u} .
$$

In the rest of the paper only mean orbital elements will be used, then in order to relieve the notation, the overline symbol is dropped.

As shown in [14], three strategies can be used to correct the elements of a satellite while maximizing the effect of the control action and minimizing the undesired change on the other elements: 1) a pair of impulses (one at apogee and one at perigee, in any order) aligned with the velocity vector can correct only the ( $a$, $e$ ) pair; 2) a single impulse (at the critical argument of true latitude) aligned with the angular momentum vector can correct only the $(i, \Omega)$ pair; 3 ) a pair of impulses (one at apogee and one at perigee, in any order) aligned with the apoaxis can correct only the $(\omega, / R)$ pair. The interest in correcting $a, e$ and $i$ pushed then towards the investigation of the first and the second strategy which from hereon, due to the direction of the applied thrust, will be denoted respectively as IPM (In-Plane Maneuver) and OPM (Out-of-Plane Maneuver). With respect to [14], in this work the authors spread the $\Delta V$ required by each impulse over an arc of trajectory. Denoting the locations where the impulses should be provided if the maneuvers were impulsive as nominal points, it is trivial that during a burning arc the satellite moves and that the 
optimal conditions (which hold at those particular points) are not held for the entire thrusting time. The overall result is that at the end of the control action small differences between the desired and the current value of the elements can be experienced; throughout the paper these differences are denoted as errors. The spreading action is performed in a way similar to what was done in [11], but with the difference that the 2 sub-arcs separated by the nominal point could have different lengths. To limit the previously cited errors it is necessary to limit the maximum deviation from the optimal condition, by imposing a maximum value for the firing time.

\section{Presentation of the Control Strategy}

To introduce the developed strategy, let us start having a look at the path followed by the satellite, from the computation until the execution of a control action. In Fig. 1 several points and arcs can be distinguished. Point $O$ denotes the origin, i.e. the position where the change of the orbital elements is noticed, the maneuvers are computed and the data are stored on-board. Starting from point $O$, the satellite follows a sequence of coasting and burning arcs (colored in black and blue respectively) and reaches the final point $E$ where the correction of the orbital elements is completed. The black point $M$ denotes the nominal point which is located approximately at the center of the burning arc, the blue points $B$ and $E$ highlight the beginning and the end of the control action and the red point $C$ represents the check point, i.e. the locations before the beginning of the control action where the maneuver strategy is checked. As a matter of fact it may happen that at point $O$ the required maneuver is wrongly computed, due for example to errors in the measurements. In such a case the mistake can be noticed and the correction can be provided on time.

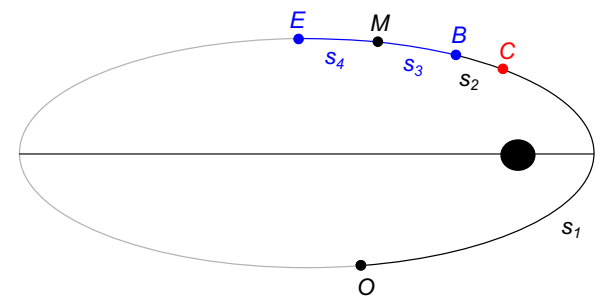

Fig. 1 Control action scheme with overview of the required points and trajectory arcs.

At the beginning of the computation it is supposed that the current mean elements $\mathbf{e}_{O}$ as well as the changes to be applied $\Delta \mathbf{e}$ are known. The maneuver strategy (i.e. the location of the points, the length of the arcs, the consumed mass, etc.) is evaluated through the resolution of a system of nonlinear equations obtained by constraining, for each point and for each element $\propto$, the match of the element values before the point and after the point. Inside a segment the element $\propto$ evolves according to the equations of motion, which always include the natural dynamics 
and in the burning arcs are properly adapted to take into account the effect of the control action.

Given two subsequent times $t_{1}$ and $t_{2}$, the variation of the element $\propto$ can always be computed as

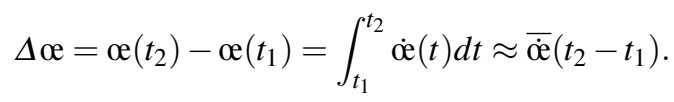

where $\overline{\dot{\alpha}}=0.5\left(\dot{\mathrm{e}}\left(t_{1}\right)+\dot{\mathfrak{e}}\left(t_{2}\right)\right)$ is an average value of the element rate within the considered time interval. Due to the slow variation of the secular rates of change of the orbital elements, the approximation introduced in Eq. (6) can be applied lightening then the complexity of the model and the computational burden which would be associated to the evaluation of the integral term.

This basic procedure is applied in each arc and for each orbital element, then expressions on the example of Eq. (6) are detected and used as constraints to evaluate the maneuver strategy. The expressions describing the rate of the mean orbital elements required to develop such constraints can be found in [18].

With reference to Fig. 1, given two generic subsequent points $X$ and $Y$ and the enclosed arc $s_{\xi}$, for the mean element $\propto$ it can be shown that

$$
\varlimsup_{Y}=œ_{X}+\Delta \varlimsup_{N}+\Delta œ_{C}
$$

where $\Delta œ_{N}$ and $\Delta œ_{C}$ denote the variation of œ due to natural dynamics and control action, respectively. When these terms are given explicitly and Eq. 7 is written for each $\propto$, it expands into

$$
\begin{aligned}
a_{Y} & =a_{X}+\dot{a}_{\xi} \Delta t_{\xi}+\delta_{l} \Delta a_{\xi} \\
e_{Y} & =e_{X}+\dot{e}_{\xi} \Delta t_{\xi}+\delta_{l} \Delta e_{\xi} \\
i_{Y} & =i_{X}+\dot{i}_{\xi} \Delta t_{\xi}+\delta_{l} \Delta i_{\xi} \\
\Omega_{Y} & =\Omega_{X}+\dot{\Omega}_{\xi} \Delta t_{\xi}+\delta_{l} \Delta \Omega_{\xi} \\
\omega_{Y} & =\omega_{X}+\dot{\omega}_{\xi} \Delta t_{\xi}+\delta_{l} \Delta \omega_{\xi} \\
\pi_{Y} & =I H_{X}+\dot{I}_{\xi} \Delta t_{\xi}+\delta_{l} \Delta / \pi_{\xi}
\end{aligned}
$$

which is coupled with the following expressions obtained from the GVE: 


$$
\begin{aligned}
\Delta a_{\xi} & =2\left(1 \pm e_{\xi}\right) \frac{\sqrt{a_{\xi}^{3}}}{\sqrt{\mu} \sqrt{1-e_{\xi}^{2}}} \Delta v_{\xi, \theta} \\
\Delta e_{\xi} & = \pm 2 \frac{\sqrt{p_{\xi}}}{\sqrt{\mu}} \Delta v_{\xi, \theta} \\
\Delta i_{\xi} & =\frac{r_{\xi}}{h_{\xi}} \cos \theta_{\xi} \Delta v_{\xi, h} \\
\Delta \Omega_{\xi} & =\frac{r_{\xi}}{h_{\xi}} \frac{\sin \theta_{\xi}}{\sin i_{\xi}} \Delta v_{\xi, h} \\
\Delta \omega_{\xi} & =\mp \frac{\sqrt{p_{\xi}}}{e_{\xi} \sqrt{\mu}} \Delta v_{\xi, p}-\Delta \Omega_{\xi} \cos i_{\xi} \\
\Delta / \pi_{\xi} & = \pm \frac{\sqrt{a_{\xi}}\left(1 \mp e_{\xi}\right)^{2}}{e_{\xi} \sqrt{\mu}} \Delta v_{\xi, p}
\end{aligned}
$$

In the above systems of constraints (8) and (9), it holds that:

- $\alpha_{k}$ denotes...

... the value of the parameter $\alpha$ at point $k$, if $k \in\left[\begin{array}{ll}X & Y\end{array}\right]$

$\ldots$ an averaged value of the parameter $\alpha$ within the arc $s_{k}$, if $k=\xi$

- $\Delta \alpha_{\xi}$ denotes...

... the variation of the element $\alpha$ due to the control action, if $\alpha \in[$ a e i $\Omega \omega / \mathrm{H}]$ ... the duration of the arc, if $\alpha=t$

$\ldots$ the required $\Delta V$, if $\alpha=v$; the addition of a $p$, a $\theta$ or an $h$ at the end of the subscript is used to highlight the direction of the velocity component

- $\dot{\alpha}_{\xi}$ denotes the natural rate of change of the element $\alpha$

- $\delta_{l}$ denotes the Kronecker $\delta$ defined as

$$
\delta_{l}=\left\{\begin{array}{l}
0: \text { if } l \text { is a coasting arc } \\
1: \text { if } l \text { is a burning arc. }
\end{array}\right.
$$

Double signs in system (9) are associated to the location of the burning arcs: the upper/lower sign is used for the arcs near the perigee/apogee.

The complete system of nonlinear equations to be solved can be finally retrieved writing Eq. 8 and 9 for all of the arcs and including the additional constraints

$$
\Delta œ=\sum_{\xi} \Delta œ_{\xi} \quad \forall œ
$$

which ensures that for each element $\propto$, the total variation obtained through the different control actions equals the desired change.

When deducing system (9) a source of error has been introduced, since the $\sin (\boldsymbol{H})$ and $\cos (\boldsymbol{A})$ terms of Eqs. (1a, 1b, 1e, 1f) have been replaced with 0,1 or -1 . This substitution is actually valid when the satellite is at the apogee/perigee and there would 
be no associated errors if the maneuvers were impulsive [14], since they would be performed exactly at the apogee/perigee. Conversely in the proposed strategy the cited trigonometric terms change with time, because the maneuver is spread along a burning arc around the nominal point. Given the requirement of limiting the maximum burning time, the maximum $\Delta A$ wrt to the apogee/perigee is limited as well, then the proposed approximation is still acceptable. The only drawback is that, as already mentioned, small errors can be experienced.

Leaving the sine and cosine terms in (9) would probably lead to more accurate results, but the computational burden associated to the resolution of the system of nonlinear equations would be higher. For this reason this option has been excluded.

One last remark concerns the direction of the thrust, which has been kept constant (in the $S p \theta h$ frame) in each control action, in order to improve the fidelity of the maneuvers.

The system obtained combining Eqs. (8), (9) and (10) represents the general formulation of the problem and must be verified for each pair of subsequent points $X$ and $Y$ of the path followed by the spacecraft. To develop a particular strategy, the problem needs to be customized using the required elements correction $\Delta \mathbf{e}$ with the proper sequence of points and term $\delta_{l}$.

\subsection{OPM Strategy}

The OPM strategy is used to correct $i$ through a single out-of-plane control action,

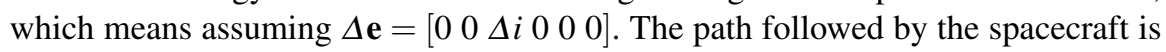
then exactly the path represented in Fig. 1, requiring 2 coasting arcs, 2 burning arcs and a sequence of 5 points $S_{O P M}=[O C B M E]$. To determine the OPM strategy, the system given by Eqs. (8), (9) and (10) must be verified for each pair of subsequent points $X$ and $Y$ separated by an arc $s_{\xi}$ with $(X, Y) \in S_{O P M}$ and $\xi \in[1,4]$. The burning arcs can be distinguished from the coasting ones through the term $\delta_{l}$, which is given by

$$
\delta_{l}=\left\{\begin{array}{l}
0: \text { if } l \in\left[\begin{array}{ll}
1 & 2
\end{array}\right] \\
1: \text { if } l \in\left[\begin{array}{ll}
3 & 4
\end{array}\right]
\end{array} .\right.
$$

\subsection{IPM Strategy}

The IPM strategy corrects $a$ and/or $e$ and consists of two separated in-plane control actions, occurring one at the apogee and one at the perigee, in any order. The chance to outline a single control action as 2 burning arcs following 2 coasting arcs leads to an IPM overall path composed by 4 coasting arcs and 4 burning arcs; this path is pictured in Fig. 2 in a manner similar to what was shown in Fig. 1. The subscripts 1 and 2 associated to the points $[C B M E]$ denote then the number of the control 
action. Please note that Fig. 2 refers to the case where the first nominal point $M_{1}$ is the perigee and the second one $M_{2}$ is the apogee; nonetheless the opposite case with $M_{1}$ as apogee and $M_{2}$ as perigee remains possible if the origin $O$ would be located after the perigee and before the apogee.

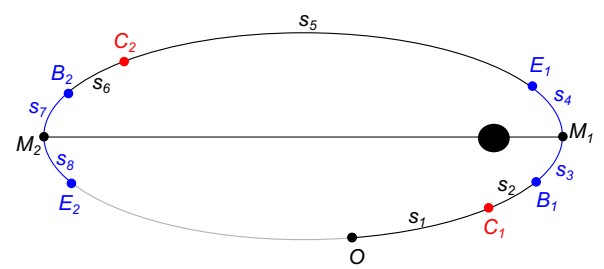

Fig. 2 IPM scheme with overview of the required points and trajectory arcs.

In the IPM case, the system obtained combining Eqs. (8), (9) and (10) must be

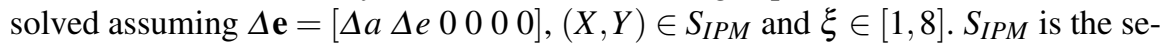

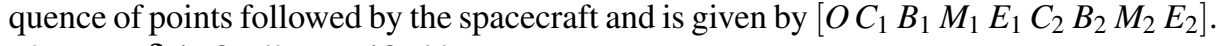
The term $\delta_{l}$ is finally specified by:

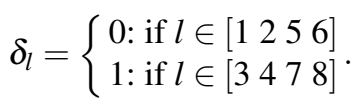

\subsection{Results Validation}

The results obtained in each strategy has been validated integrating the equations of motion of the satellite in cartesian coordinates. Through the resolution of the system of nonlinear equations the entire control scheme is determined and all the maneuver-related parameters (the orbital elements of the points, the lenght of the arcs, the consumed masses, the required $\Delta V$, the direction of the thrust, etc.) are known. The duration of the arcs and the direction of the thrust are then passed to the integrator, which evaluate the motion from the current position $O$ until the execution of the last control action. For each burning arc the variation of the orbital elements provided by the thrust is retrieved accurately excluding the contribution due to the natural dynamics. Then the real overall variations $\Delta \mathbf{e}_{R}$ obtained with all the control actions are compared with the desired changes $\Delta \mathbf{e}$ and the errors are quantified.

\section{Numerical Simulations}

For both types of maneuver (OPM and IPM), the computed strategy has been verified integrating the equations of motion in cartesian coordinates. The model used for the propagation of the satellite motion includes the perturbation of the geopotential 
and of the atmospheric drag. Regarding the geopotential only the even zonal terms have been considered, since they are the only ones influencing the mean orbital elements, while tesseral and sectorial terms affect only the short-term and the long-term variations in the osculating elements [4]. For what concerns the drag perturbation, an exponential model for the atmospherical density has been assumed.

Due to the transformations and approximations involved in the problem (transformation between osculating and mean elements, use of mean elements in the GVE, etc.) it is expected that at the end of a given maneuver the desired changes $\Delta \mathbf{e}$ will be affected by small errors. For this reason, the two types of maneuver have been treated separately and tests in a Monte-Carlo fashion have been conducted to relate the obtained errors to the desired $\Delta \mathbf{e}$.

In agreement with the proposed mission scenario, which envisages the construction of a giant structure through multiple rendez-vous and dockings of separate spacecraft, the involved agents are expected to have masses and volumes on the order of tens of tonnes and hundreds of cubic meters, respectively. Hence they have been modeled on the ATV [7] with a mass of $m=30 \mathrm{t}$ and a cylindrical shape with base diameter $d=5 \mathrm{~m}$ and side length $l=15 \mathrm{~m}$. Finally it has been guessed $C_{D}=2.2$. Each maneuver can be performed using 4 thrusters, each characterised by a nominal thrust $T=220 \mathrm{~N}$, specific impulse $I_{s p}=270.22 \mathrm{~s}$ and minimum impulse bit $M I B=8$ Ns.

In the following at first an example for OPM and one for IPM are presented to compare the unperturbed and the perturbed cases, then the Monte-Carlo simulation campaign is discussed.

\subsection{Maneuver Examples}

In this section an example for the OPM and one for the IPM is presented. For each type of maneuver, 4 cases have been studied to compare the effect of the different perturbations:

1. keplerian motion

2. zonal terms up to $J_{2}$

3. zonal terms up to $J_{6}$

4. zonal terms up to $J_{6}$ and atmospheric drag.

\subsubsection{OPM Example}

For the illustration of the OPM the osculating elements given in Table 1 denote the initial conditions, i.e. the state of the satellite at point $O$. This is the location where the need for an inclination change $\Delta i=-0.0582 \mathrm{deg}$ is noticed.

From the computation of the maneuver it results that the thrusters are activated for approximately $262.51 \mathrm{~s}$, providing a $\Delta V=7.7114 \mathrm{~m} / \mathrm{s}$ and consuming a mass of propellant $m_{p}=87.1726 \mathrm{~kg}$. 
Table 1 Satellite initial osculating elements for the OPM

\begin{tabular}{crc}
\hline Element & \multicolumn{1}{c}{ Value } & Units \\
\hline$a$ & 7000.8469 & $\mathrm{~km}$ \\
$e$ & 0.0011 & \\
$i$ & 50.5583 & $\mathrm{deg}$ \\
$\Omega$ & 87.4406 & $\mathrm{deg}$ \\
$\omega$ & 119.2945 & $\mathrm{deg}$ \\
$\boldsymbol{I} \boldsymbol{H}$ & 275.3817 & $\mathrm{deg}$ \\
\hline
\end{tabular}

A comparison of the 4 considered cases showed very tiny differences in the obtained solutions. Due to the limited short duration of the maneuver, the inclusion of the perturbation has no perceivable effect on parameters like the $\Delta V$, the firing time and the consumed mass. The perturbing actions affects instead the waiting time, i.e. the time required to reach the location where the thrusters are turned on. However, even for this parameter, the biggest difference is on the order of few seconds and can be seen between case 1 and case 2, since it is due, as expected, to the $J_{2}$ term. Differences between cases 2, 3 and 4 are less than a hundredth of a second. Table 2 summarizes the waiting times $\Delta t_{w}$ and the firing times $\Delta t_{f}$ for each of the 4 considered cases. The evolution of the elements is shown in Fig. 3 for Case \#2.

Table 2 Results of the OPM Example

\begin{tabular}{cccccc}
\hline Parameter & Case 1 & Case 2 & Case 3 & Case 4 & Units \\
\hline$\Delta t_{w}$ & 2216.2402 & 2214.2967 & 2214.2985 & 2214.2985 & $\mathrm{~s}$ \\
$\Delta t_{f}$ & 262.5086 & 262.5100 & 262.5100 & 262.5100 & $\mathrm{~s}$ \\
\hline
\end{tabular}

\subsubsection{IPM Example}

For this example the state of the satellite at point $O$ is given in Table 3 . The desired semimajor axis and eccentricity changes are $\Delta a=-7.9824 \mathrm{~km}$ and $\Delta e=0.0013$.

The transfer strategy is composed by two maneuvers requiring an overall $\Delta V$ of $5.0508 \mathrm{~m} / \mathrm{s}$, which translates in $57.1252 \mathrm{~kg}$ of propellant. The first maneuver is performed at time $t_{1} \approx 1150.53 \mathrm{~s}$ and lasts for $\Delta t_{f, 1}=12.61 \mathrm{~s}$, the second one takes place at time $t_{2} \approx 3995.14 \mathrm{~s}$ and lasts for $\Delta t_{f, 2}=159.41 \mathrm{~s}$.

Similarly to Sect. 4.1.1, the results are presented in Table 4, showing that the effect of the perturbations can be seen only on the waiting times. Fig. 4 provides an overview of the elements evolution from Case \#2. 


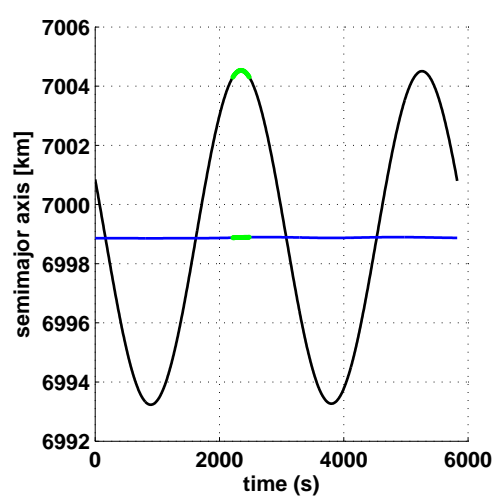

(a)

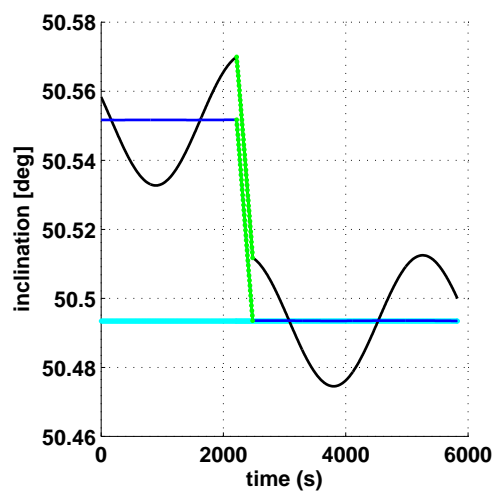

(c)

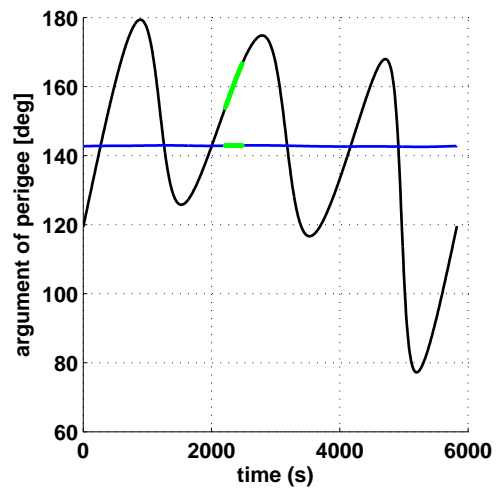

(e)

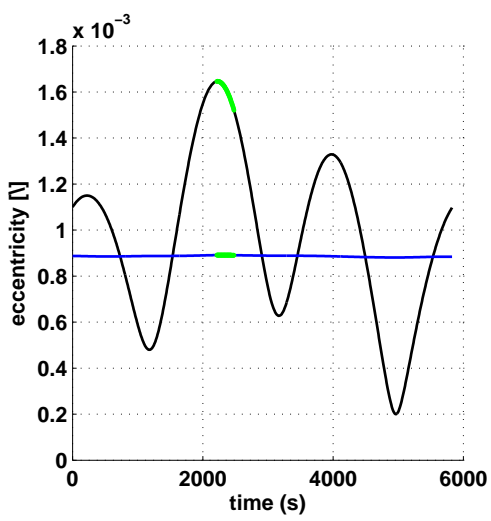

(b)

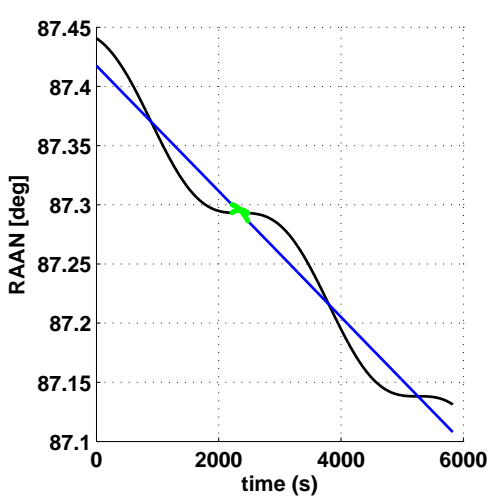

(d)

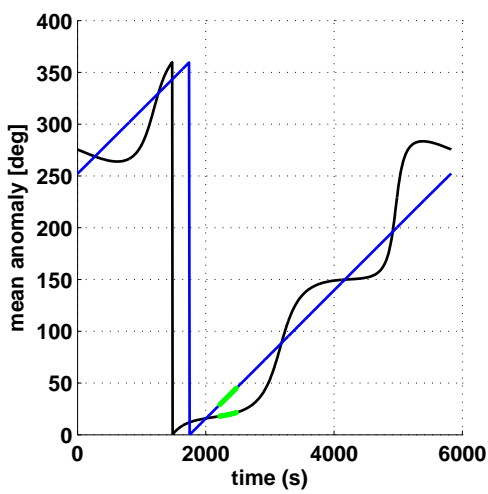

(f)

Fig. 3 Element evolutions in the OPM example (case \#2). In each subfigure the blue and the black lines represent the osculating and the mean element, respectively. The green line marks the burning arc. The cyan line highlights the new mean inclination that should be achieved with the control action. 


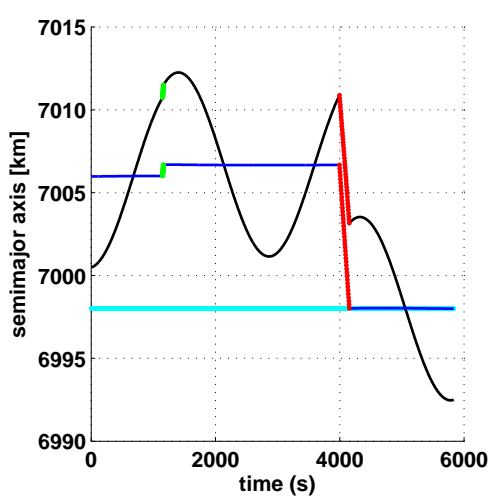

(a)

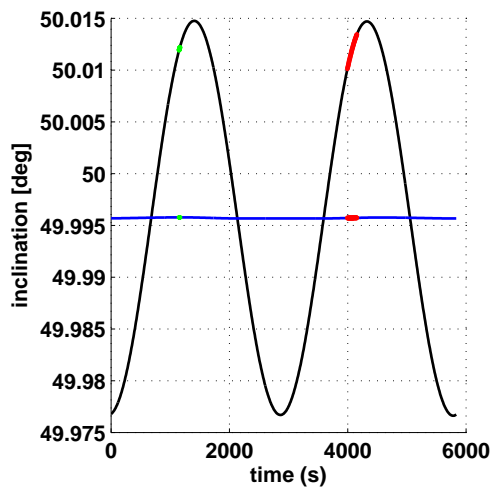

(c)

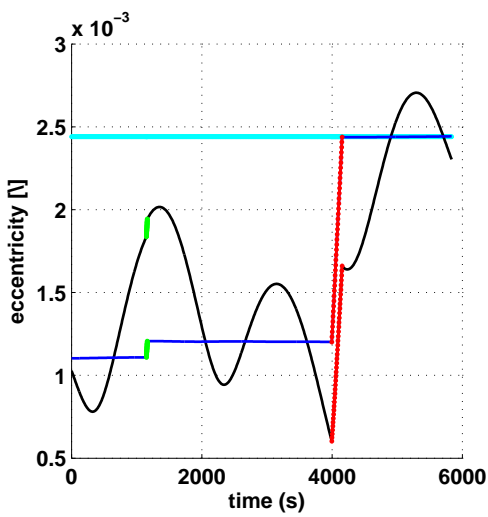

(b)

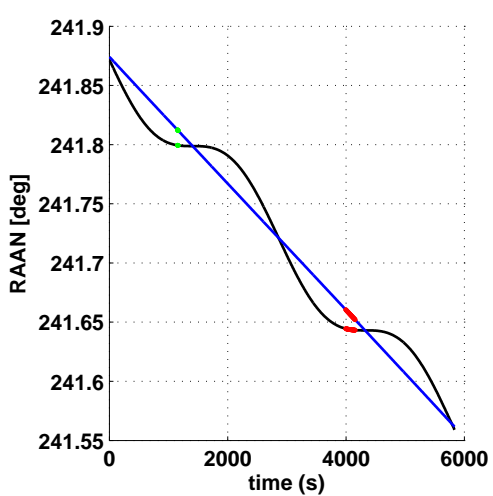

(d)

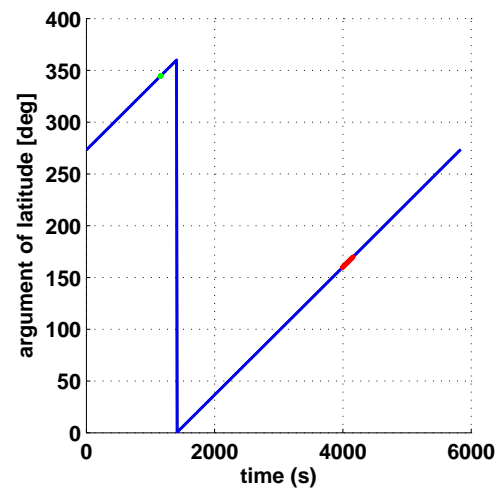

(e)

Fig. 4 Element evolutions in the IPM example (case \#2). In each subfigure the blue and the black lines represent the osculating and the mean element, respectively. The green and the red lines correspond to the burning arcs. The cyan lines highlight the new semimajor axis and eccentricity that should be achieved with the control actions. 
Table 3 Satellite initial osculating elements for the IPM

\begin{tabular}{crc}
\hline Element & \multicolumn{1}{c}{ Value } & Units \\
\hline$a$ & 7000.4828 & $\mathrm{~km}$ \\
$e$ & 0.0010 & \\
$i$ & 49.9767 & $\mathrm{deg}$ \\
$\Omega$ & 241.8717 & $\mathrm{deg}$ \\
$\omega$ & 10.9910 & $\mathrm{deg}$ \\
$\Gamma \boldsymbol{H}$ & 261.9797 & $\mathrm{deg}$ \\
\hline
\end{tabular}

Table 4 Results of the IPM Example

\begin{tabular}{cccccc}
\hline Parameter & Case 1 & Case 2 & Case 3 & Case 4 & Units \\
\hline$\Delta t_{w, 1}$ & 1150.7225 & 1150.5358 & 1150.5357 & 1150.5357 & $\mathrm{~s}$ \\
$\Delta t_{f, 1}$ & 12.6126 & 12.6126 & 12.6126 & 12.6126 & $\mathrm{~s}$ \\
$\Delta t_{w, 2}$ & 2832.3900 & 2831.9193 & 2831.9190 & 2831.9189 & $\mathrm{~s}$ \\
$\Delta t_{f, 2}$ & 159.4123 & 159.4123 & 159.4123 & 159.4123 & $\mathrm{~s}$ \\
\hline
\end{tabular}

\subsection{Monte-Carlo Results}

The Monte-Carlo campaign of simulations has been conducted to relate the undesired errors obtained at the end of a maneuver with the desired mean elements variations that the maneuver should provide. In addition, from the obtained results, an overview of the maneuvers attainable with the given propulsion system is available.

In order to limit the undesired errors due to the spreading of the $\Delta V$, a maximum duration of 5 minutes has been assumed for each firing action. Similarly, a minimum firing time of approximately $0.03 \mathrm{~s}$ has been imposed according to the MIB.

The minimum firing time has actually a double function, since it can be indirectly used to distinguish between cyclic and non-cyclic solutions. In the performed analysis, when the magnitude of the errors are above a certain threshold, the solution is called cyclic because a new maneuver is required to correct those errors, with the overall result that a longer time is required to complete the process. On the other side, when the errors do not exceed the threshold, the solution is non-cyclic and no additional corrections are required. Hence, to develop this distinction the threshold must be defined. To this end, once the propulsion system (and then its thrust level) is known, the GVE can be easily used to estimate the change in orbital elements which would be achieved for a given firing time. The minimum changes (i.e. the changes associated to the minimum firing time) can then be used as threshold for the maximum acceptable errors $E_{\max }$, since it is trivial that changes below these values cannot be achieved. In this study, there were no constraints on the total time required to complete the maneuver, nonetheless the authors decided to keep this parameter bounded, then the attention has been directed only towards the non-cyclic solutions, while the cyclic ones have been considered unacceptable. 
One last remark concerns the included perturbations. Some preliminary tests, as well as the examples shown in Sect. 4.1.1 and 4.1.2, showed that the inclusion of high order zonal terms and atmospheric drag produces no significant differences in the obtained results. For this reason, in order to lighten up the computation burden, in the Monte-Carlo campaigns only the $J_{2}$ term has been used to perturb the motion.

\subsubsection{Simulation Campaign for OPM}

In this first campaign of tests, OPMs to change only the inclination by an amount $\Delta i$ have been simulated. In each simulation a different $\Delta i$, as well as a different set of initial conditions $\mathbf{e}_{O}$, has been used. In building the vector $\mathbf{e}_{O}$ the last elements $\Omega, \omega$

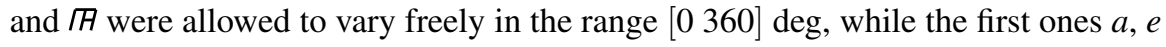
and $i$ were picked in a neighbourhood of the nominal values. Concerning the desired $\Delta i$, the considered values are both positive and negative, with the maximum value (in magnitude) depending on the maximum firing duration requirement. Results are presented in Fig. 5, where each subfigure is dedicated to a different orbital element. On the $x$-axis there is the change $\Delta i$ to be applied, while the $y$-axis shows the error of the considered mean element. Please note that Fig. 5(c) shows a percentage error, since the error obtained at the end of the maneuver is compared with the desired change. Starting the analysis with this subfigure, at first a reasonable threshold of $1 \%$ has been assumed valid, but afterwards the results showed that the obtained errors are even 1 order of magnitude smaller, then no constraints are imposed by the inclination error. Conversely, from the other subfigures, in which the horizontal red lines represent the maximum acceptable error $E_{\max }$, it can be seen that only when $|\Delta i|$ is below a certain threshold the maneuver is always non-cyclic and then acceptable. The $|\Delta i|$ thresholds associated to the different elements are shown in the second column of Table 5 . The bar $(\backslash)$ in the third row marks the fact that no constraints must be imposed on $\Delta i$ to keep the inclination error under the maximum value $E_{\max }$. Using again the GVE, for each element the correspondent $|\Delta i|$ threshold can be associated to a time interval representing the maximum firing time over which the element error exceeds the threshold and the maneuver could be cyclic. The six time values can be retrieved in the last column of Table 5 .

From Table 5 it can be seen that the maximum times are not equal for all the elements. For example after a $100 \mathrm{~s}$ control action, the errors on $a, e$ and $i$ would be below the maximum acceptable error, but nothing could be said for the remaining elements $\Omega, \omega$ and $/$. For this reason, to guarantee that all of the final errors are acceptable, the maximum duration of an OPM should not be longer than $17 \mathrm{~s}$, which is the minimum among the six times in Table 5: a maneuver shorter than $17 \mathrm{~s}$ always produces errors small enough to be neglected, a maneuver longer might produce errors big enough to require a new control action for canceling them. 


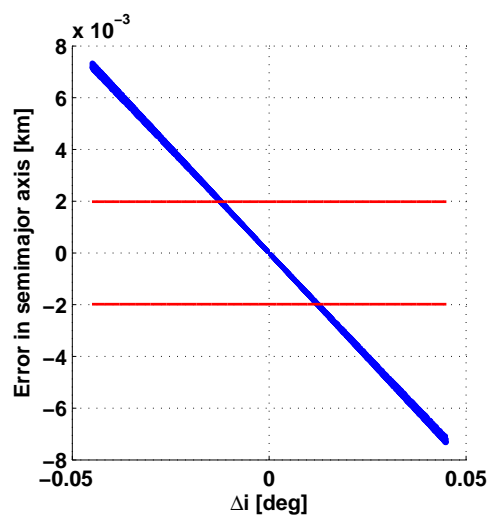

(a)

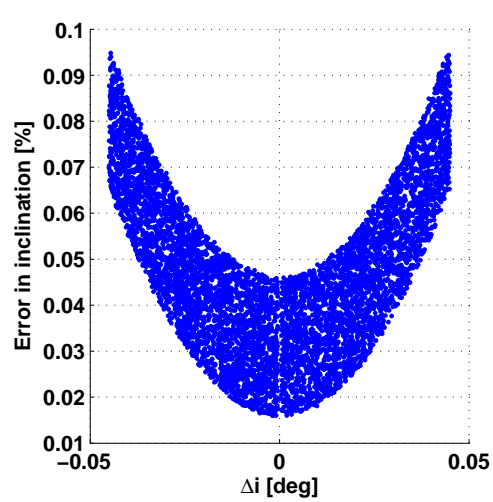

(c)

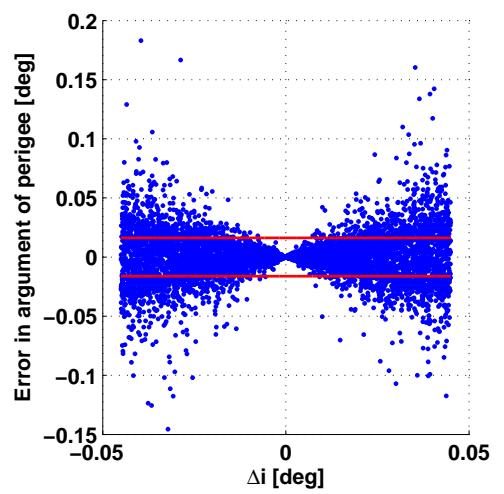

(e)

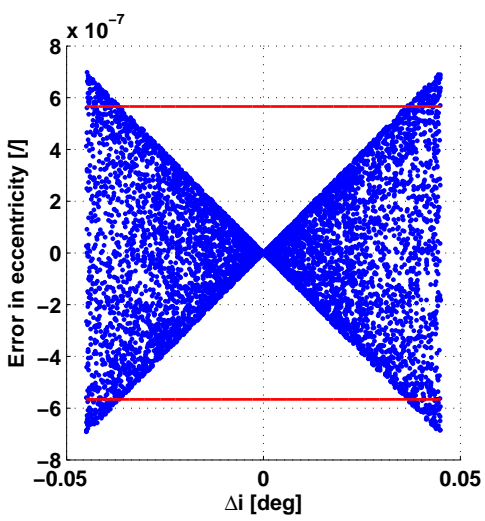

(b)

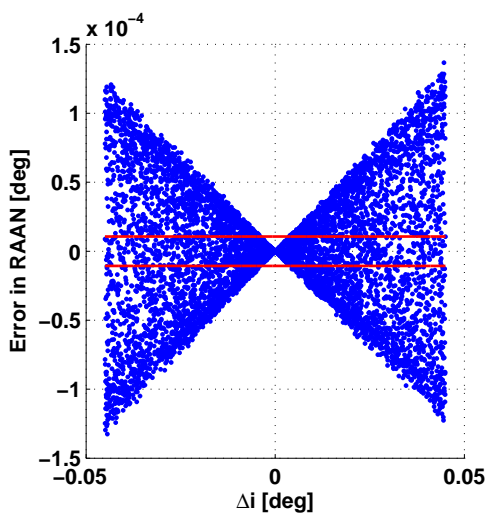

(d)

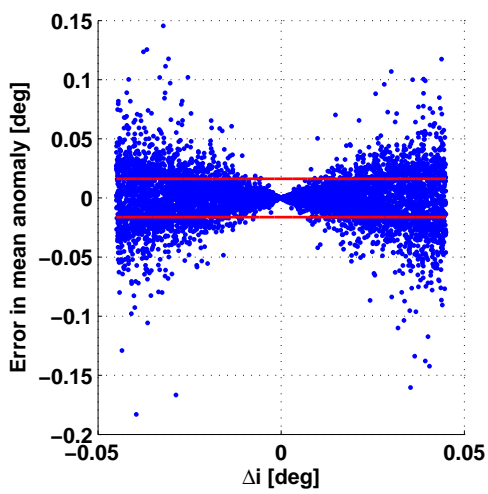

(f)

Fig. 5 Mean elements errors at the end of the OPM strategy: (a) semimajor axis (b) eccentricity (c) inclination (d) longitude of the ascending node (e) argument of perigee (f) mean anomaly. 
Table 5 Approximate error and time thresholds for the OPM

\begin{tabular}{ccc}
\hline Element & $|\Delta i|[\mathrm{deg}]$ & Time $[\mathrm{s}]$ \\
\hline$a$ & 0.024 & 108 \\
$e$ & 0.037 & 167 \\
$i$ & $\backslash$ & 300 \\
$\Omega$ & 0.003 & 17 \\
$\omega$ & 0.005 & 24 \\
$\Gamma \boldsymbol{H}$ & 0.005 & 24 \\
\hline
\end{tabular}

\subsubsection{Simulation Campaign for IPM}

The second series of tests dealt with IPMs to change semimajor axis and eccentricity by an amount $\Delta a$ and $\Delta e$. Similarly to what has been done for the OPM case, each simulation is characterised by a different pair $(\Delta a, \Delta e)$ and a different set of initial conditions $\mathbf{e}_{O}$. Results are presented in Fig. 6, with each subfigure associated to a different orbital element. The $x$-axis is associated to $\Delta a$, the $y$-axis to $\Delta e$ and the $z$ axis to the error obtained at the end of the maneuver. For a matter of clearness, this time the error thresholds are not plotted. Moreover, since an in-plane applied $\Delta V$ has a stronger impact than an out-of-plane one on $\omega$ and $/$, the errors on these elements are now bigger. Nonetheless they usually have the same order of magnitude and opposite sign, with the consequence that the argument of latitude is almost zero. For this reason, in Fig. 6 the error on the argument of latitude replaced the errors on argument of perigee and mean anomaly.

To better understand which $(\Delta a, \Delta e)$ pairs can produce non-cyclic solutions, all the subfigures from Fig. 6 have been projected and analysed in the $x y$-plane. The final result is given in Fig. 7. All the tests used a $(\Delta a, \Delta e)$ pair inside the external black rectangle, but only those inside the green bounded area lead to non-cyclic maneuvers and can be considered acceptable. When the analysis is based on $\omega$ and I 7 instead of $\theta$, the acceptable area shrinks considerably and non-cyclic maneuvers can be obtained only using $(\Delta a, \Delta e)$ pairs inside the blue bounded region.

\section{Conclusions}

An approach to compute quasi-impulsive maneuvers aimed at steering the mean orbital elements of a spacecraft to a desired value has been presented. When performing a transfer to change some of the spacecraft orbital elements, sometimes it is enough to achieve the desired values neglecting the undesired changes obtained on the other elements. Nevertheless there are cases where those changes are unacceptable and then particular attention must be paid on the maneuver strategy.

In this paper two strategies have been studied to provide small changes in semimajor axis and eccentricity or in inclination while minimizing the errors on the other 


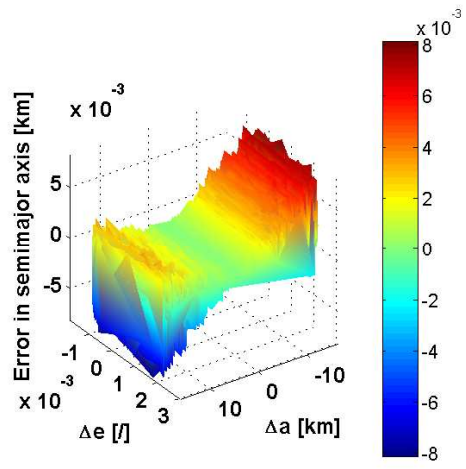

(a)

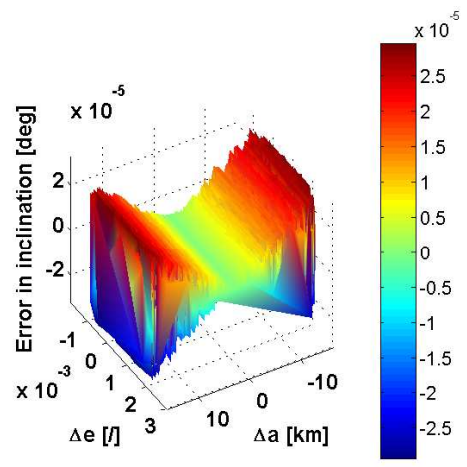

(c)

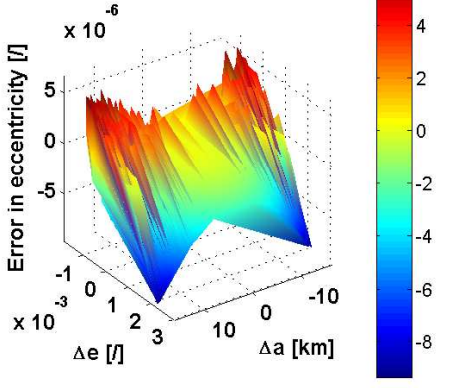

(b)

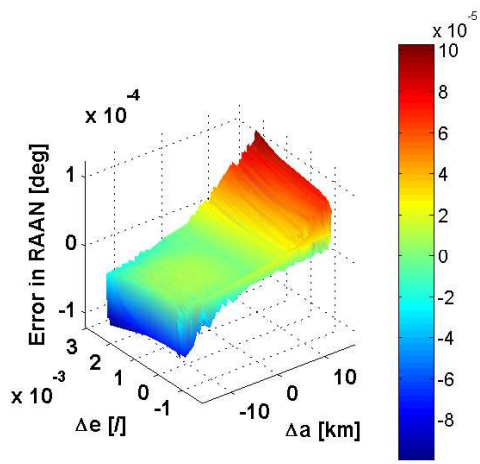

(d)

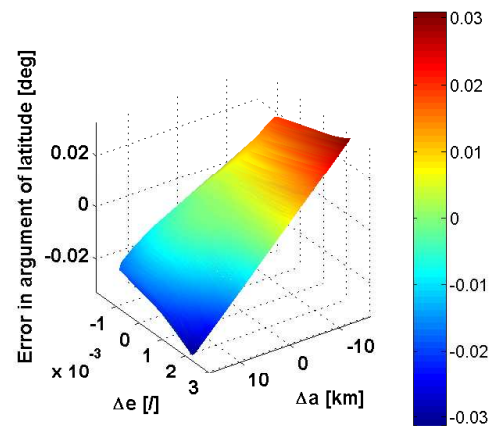

(e)

Fig. 6 Mean elements errors at the end of the IPM strategy: (a) semimajor axis (b) eccentricity (c) inclination (d) longitude of the ascending node (e) argument of latitude. 


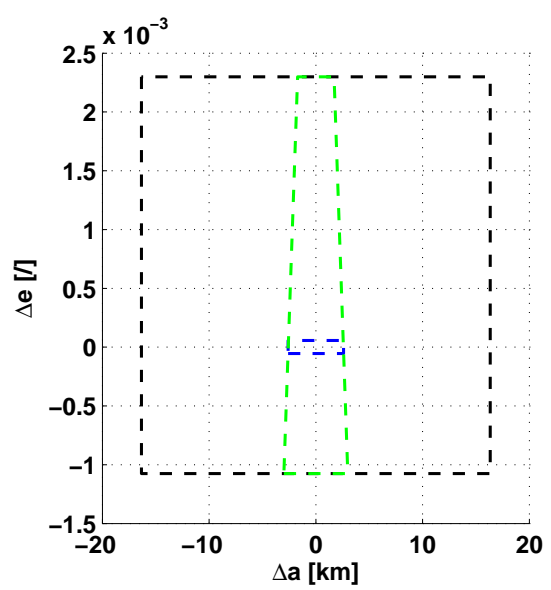

Fig. 7 Map of $(\Delta a, \Delta e)$ pairs acceptability

elements. These errors are difficult to predict due to the different sources on which they depend, then wide campaigns of simulations have been performed to use the statistical approach for better understanding the behaviour of the problem.

Example tests have been presented and discussed for each strategy.

The inclusion of the zonal terms of the gravity model and of the atmospheric drag guarantee high accuracy of the model. The obtained results showed that no big differences can be seen in the thrust duration and direction, but a better evaluation of the maneuvering location can be achieved.

The knowledge of the propulsion system parameters allows an high fidelity maneuver simulation, inasmuch as any required $\Delta V$ is spread along an arc of the trajectory. The corresponding control actions have then a finite duration, but the small changes in the orbital elements keep them short enough to accept some simplifying assumptions typically used in the impulsive method. The presented quasi-impulsive approach results then from this combination of the impulsive and the finite ones.

Due to the assumed models, the obtained changes $\Delta \mathbf{e}_{R}$ do not match perfectly the desired changes $\Delta \mathbf{e}$ and some errors can be experienced. Comparing these errors with some reference thresholds, it is possible to distinguish between acceptable solutions (the errors are below the thresholds) and unacceptable solutions (the errors are above the thresholds and require a new maneuver to be canceled). Since the magnitude of these errors grows with the magnitude of the desired changes, a range of $\Delta \mathbf{e}$ guaranteeing acceptable solutions can be identified.

Acknowledgements The authors wish to thank their colleague Dr Stephen Steffes for the valuable comments and criticisms provided, especially in the review of the manuscript. 
Quasi-Impulsive Maneuvers to Correct Mean Orbital Elements in LEO

\section{References}

1. Anderson PV, Schaub H (2012) Impulsive feedback control of nonsingular elements in the geostationary regime. AIAA/AAS Astrodynamics Specialist Conference, Minneapolis, Minnesota, paper AIAA 2012-4585

2. Battin RH (1999) An introduction to the mathematics and methods of astrodynamics, revised edition. AIAA Education Series. ISBN: 9781600860263

3. Beigelman I, Gurfil P (2009) Optimal geostationary collocation using relative orbital element corrections. Journal of Spacecraft and Rockets 46(1):141-150

4. Blitzer L (1970) Handbook of orbital perturbations. Astronautica 453, University of Arizona.

5. Broucke RA, Prado AFBA (1994) Optimal N-impulse transfer between coplanar orbits. AAS/AIAA Astrodynamics Specialist Conference, Victoris, Canada, 16-19 Aug. 1994, paper AAS 93-660

6. Eckel KG, Nguyen XV (1984) Optimal switching conditions for minimum fuel fixed time transfer between non coplanar elliptical orbits. Acta Astronautica 11(10/11):621-631

7. ESA (2007) Automated Transfer Vehicle (ATV), ESA document EUC-ESA-FSH-003 Rev. 1.2 (specifications)

8. Kamel OM, Soliman AS (2010) Optimum bi-impulsive non coplanar elliptic Hohmann type transfer. Mechanics and Mechanical Engineering 14(1):81-104

9. Lawden DF (1992) Optimal transfers between coplanar elliptical orbits. Journal of Guidance, Control, and Dynamics 15(3):788-791

10. Mazal L, Gurfil P (2011) Close-form solution of the 11-optimal bi-impulsive coplanar orbital transfer problem. Journal of Guidance, Control, and Dynamics 34(6):1936-1940

11. Pollard JE (2000) Simplified analysis of low-thrust orbital maneuvers. The Aerospace Corporation TR-2000(8565)-10

12. Ruggiero A, Pergola P, Marcuccio S, Andrenucci M (2011) Low-thrust maneuvers for the efficient correction of orbital elements. 32nd International Electric Propulsion Conference, Wiesbaden, Germany, paper IEPC-2011-102

13. Schaub H, Vadali SR, Junkins JL, Alfriend KT (2000) Spacecraft formation flying control using mean orbit elements. Journal of Astronautical Sciences 48(1):69-87

14. Schaub H, Alfriend KT (2001) Impulsive feedback control to establish specific mean orbit elements of spacecraft formations. Journal of Guidance, Control, and Dynamics 24(4):739745

15. Schaub H, Alfriend KT (2001) $J_{2}$ invariant relative orbits for spacecraft formations. Celestial Mechanics and Dynamical Astronomy 79(2):77-95

16. Schulz W, Prado AFBA (2006) Optimal space maneuvers in three dimensions. Journal of the Brazilian Society of Mechanical Sciences and Engineering 28(4):375-377

17. Taraba M, Zwintz K., Bombardelli C, Lasue J, Rogler P, Ruelle V, Schlutz J, Schüßler M, O'Sullivan S, Sinzig B, Treffer M, Valavanoglou A, van Quickelberghe M, Walpole M, Wessels L (2006) Project $\mathrm{M}^{3}$ - a study for a manned Mars mission in 2031. Acta Astronautica 58:88-104

18. Zhong W, Gurfil P (2013) Mean orbital elements estimation for autonomous satellite guidance and orbit control. Journal of Guidance, Control, and Dynamics 36(6):1624-1641 